\title{
GPU-Based Hybrid Method for Electromagnetic Scattering of Electrically Large Objects
}

\author{
ZHU Yan-ju ${ }^{1, a}$, XIE Shu-guo ${ }^{1}$ \\ ${ }^{1}$ School of Electronic and Information Engineering, Beijing University of Aeronautics and \\ Astronautics, Beijing 100191, China \\ azhuyanju1309@163.com
}

\begin{abstract}
Keywords: Compute Unified Device Architecture(CUDA); Graphical-Electromagnetic Computing
\end{abstract} (GRECO); Graphics Processing Unit (GPU); Modified Physical Optics (MPO).

\begin{abstract}
A GPU-based parallel hybrid method is proposed to accelerate the electromagnetic scattering from electrically large complex structures in high frequency. The MPO and the GRECO hybrid method can simplify the computations of the diffraction fields of the wedges by modifying the surface-normal vector of the target to redefine the surface equivalent currents. By using GPU-based reduction operation, we design a fast parallel summing method for the hybrid integrals over illuminated triangles. The hybrid method is validated by comparing the numerical results with those obtained through the serial computing program on a central processing unit, as well as through FEKO method of moments, which showed good agreement.
\end{abstract}

\section{Introduction}

With the development of the stealth technology, electromagnetic scattering of the electrically large targets in high frequency is very important for the national defense, aerospace and remote sensing, etc.. To obtain the scattered field for the complex target, the computation and memory are in high demand. Then, asymptotic methods, e.g., based on the Pyhsical Optics (PO) method, is often applied for engineering calculation of large objects. The GRECO method was introduced in the [1] for simple and fast computation of the PO surface integral. The main objective of the algorithms is obtaining results of the radar cross section (RCS)prediction in real time for arbitrary target shapes, using a graphics workstation. However, in GRECO method, it is complex to realize the wedges scattering. So we apply the unique idea of the MPO [2] to solve the problem. In the MPO, the novel surface equivalent currents can be rebuilded by using modified surface normal vector. This simple geometrical replacement of the surface normal vectors can improves the accuracy of the GRECO to a level of the geometrical theory of diffraction (GTD). In high frequency, the integral evaluation is still one of the challenging problems, especially for the electrically large complex structure. Recently, CUDA[3] from NVIDIA which has a general-purpose parallel computing architecture is introduced to apply more and more in the fields which need a great deal scientific calculation, such as image processing[4], video decoding[5], and cryptography[6], etc. In the field of computational electromagnetics, GPU computing has gained attention for acceleration[7-10].

In this paper, the GPU-based MPO/GRECO hybrid method is proposed that makes the computations of the diffraction fields of the wedges to be an easy and efficient work. Then, The integral of the hybrid method over illuminated triangles can be obtained by parallel computing methods of GPU with CUDA.

\section{Theory}

\section{The Hybrid GRECO-MPO Method.}

In GRECO, the induced currents on the illuminated region of the scattering surface is $\vec{J}_{s}=2\left(\vec{n} \times \vec{H}^{i}\right)$. where vector $\vec{n}$ is the normal vector to the surface and $\vec{H}^{i}$ is the incident field. $\vec{J}_{s}$ is the surface currents. In MPO, the modified surface normal vectors $\vec{n}_{r}$ and $\vec{n}_{i}$ are described as follows: The $\vec{n}_{r}$ is the normal vectors of reflection field which is obtained by the observer and the 
source. The $\overrightarrow{\boldsymbol{n}}_{i}$ is the normal vectors of image field, and it is similarly defined between the observer and an image source. The $\vec{n}_{r}$ and $\vec{n}_{i}$ are made to take place of $\vec{n}$, so the surface current used in GRECO integral can be modified as follows:

$\vec{J}_{S}=2\left(\vec{n}_{r} \times \overrightarrow{\boldsymbol{H}}^{i}+\vec{n}_{i} \times \overrightarrow{\boldsymbol{H}}^{i m}\right)$.

Where the image field $\bar{H}^{i m}$ can be found by using the image theory[11]

$\vec{H}^{i m}=\vec{H}^{i}-2 \vec{n}\left(\vec{H}^{i} \cdot \vec{n}\right)$.

$\overrightarrow{\boldsymbol{H}}^{s}$ can be expressed as:

$\vec{H}^{s}=\frac{-j k e^{-j k R}}{4 \pi R} \int_{s} \vec{J}_{s} \times \vec{k}_{s} e^{j k \vec{k}_{s} \cdot \vec{r}} d s^{\prime}$.

Take(1)(2)into (3), the scattered magnetic fields is:

$\overrightarrow{H^{s}}=\frac{-j k \mathrm{e}^{-j k R_{0}}}{2 \pi R_{0}} \int\left(\vec{n}_{r} \times \overrightarrow{H^{i}}+\vec{n}_{i} \times\left[\overrightarrow{H^{i}}-2 \vec{n} \cdot\left(\overrightarrow{H^{i}} \cdot \vec{n}\right)\right]\right) \times \vec{k}_{s} \mathrm{e}^{j k \vec{k}_{s} \cdot \vec{r}} \cdot d s$

$=\frac{-j k \mathrm{e}^{-j k R_{0}}}{2 \pi R_{0}} \int\left(\vec{n}_{r} \times \overrightarrow{H^{i}} \times \vec{k}_{s}+\vec{n}_{i} \times\left[\overrightarrow{H^{i}}-2 \vec{n} \cdot\left(\overrightarrow{H^{i}} \cdot \vec{n}\right)\right] \times \vec{k}_{s}\right) \mathrm{e}^{j k \vec{k}_{s} \cdot \vec{r}} \cdot d s$.

Where $\vec{k}_{s}$ is the observation direction, $\vec{k}_{i}$ is the incidence direction, we consider the monostatic condition, Fig. 1 shows the modified surface normal vectors on a triangular facet at an observation angle. so $\vec{n}_{r}=\vec{k}_{s}=-\vec{k}_{i}$ and $\vec{n}_{i}$ becomes a tangential vector to the surface for back scattered RCS calculation, we know $\overrightarrow{\boldsymbol{E}}=\boldsymbol{\eta} \overrightarrow{\boldsymbol{H}} \times \overrightarrow{\boldsymbol{k}}_{s}$ and $\overrightarrow{\boldsymbol{k}}_{s} \times \overrightarrow{\boldsymbol{k}}_{s}=0$, it can be expressed as :

$$
\left.\vec{E}_{s}=\frac{-j k \mathrm{e}^{-j k R_{0}}}{2 \pi R_{0}} \int\left(\vec{n}_{r} \cdot \vec{k}_{s}\right) \cdot \overrightarrow{H^{i}} \times \vec{k}_{s}+\left(\vec{n}_{i} \cdot \vec{k}_{s}\right) \cdot \overrightarrow{H^{i}} \times \vec{k}_{s}-2\left(\vec{n}_{i} \cdot \vec{k}_{s}\right) \cdot \vec{n} \times \vec{k}_{s} \cdot\left(\overrightarrow{H^{i}} \cdot \vec{n}\right)\right] \mathrm{e}^{j k \vec{k}_{s} \cdot \vec{r}} \cdot d s .
$$

So we can obtain the expression of horizontal polarization and vertical polarization.

Horizontal polarization:

$$
\sigma=\frac{k^{2}}{\pi}\left|\sum_{\text {pixel }}\left(\frac{-1}{\cos \theta}-\frac{\sin \theta}{\cos \theta}\right) \exp (2 \mathrm{jkz}) \cdot \Delta s\right|^{2} \text {. }
$$

Vertical polarization:

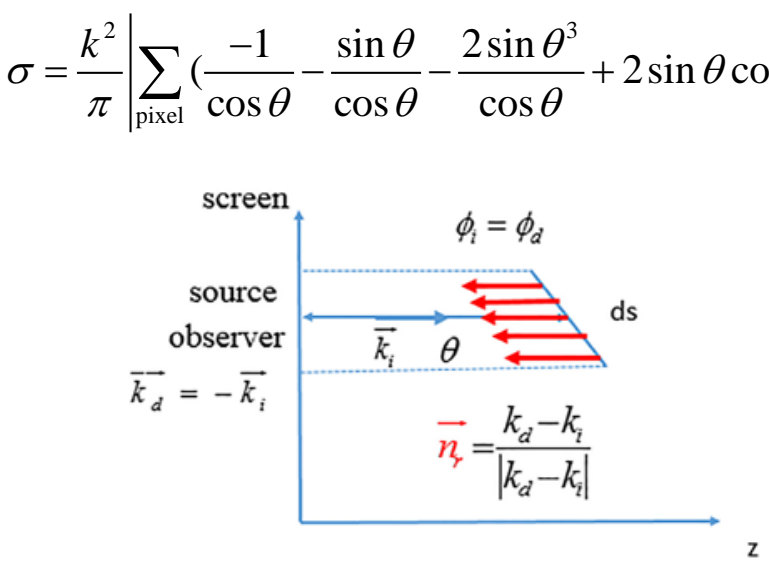

(a)

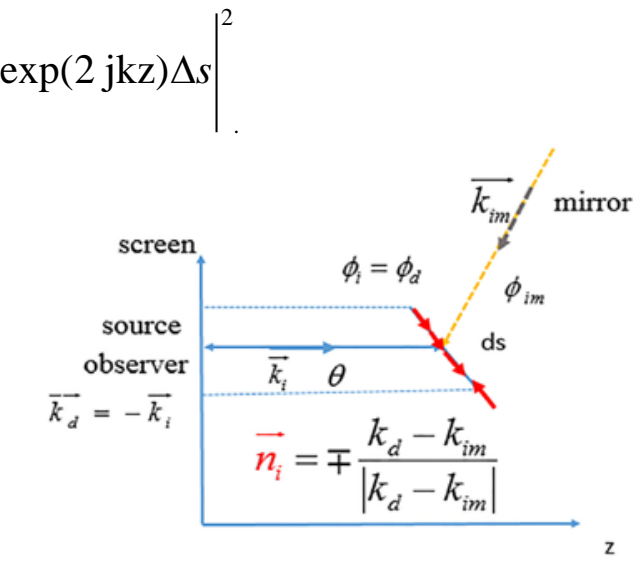

(b)

Fig. 1: The modified surface element and its projection on the work station screen $\overrightarrow{\boldsymbol{n}}_{r}$ and $\overrightarrow{\boldsymbol{n}}_{i}$.(a)

The definition of $\overrightarrow{\boldsymbol{n}}_{r}$

GPU Implementation of the Hybrid Method.

(b) The definition of $\overrightarrow{\boldsymbol{n}}_{i}$.

According to equation (6) and (7), the total hybrid method integral can be obtained by summing the divided unrelated triangles. The parallel hybrid algorithm integral computing contains two steps: one is the scattered fields computation of each triangle and the other is the scattered fields of all triangles integral summation. For each visible triangle, it can be assigned each thread to compute the scattered fields. The cluster of the threads is called a thread block. The shared memory of each thread block stores triangles integral summation. For the calculation of the total summation of each 
thread block in shared memory, it needs less time to read and write than the global memory. The parallel reduction algorithm is applied to improve efficiency, and the bank conflicts should be avoided. The integral summation of each thread block can be calculated by making the same reduction operation.

\section{Numerical simulations and discussions}

In this section, a variety of numerical examples are presented to demonstrate the accuracy and efficiency of the hybrid GRECO-MPO method based on GPU. The solutions of scattering features of the targets are realized on CPU and GPU, respectively.

Taking normal plane wave incidence with frequency $10 \mathrm{GHz}$ to $1 \mathrm{~m} * 1 \mathrm{~m}$ PEC plate(Fig. 2), and compute the RCS of the plate. The HH-polarized monostatic radar cross section (RCS) is computed by using CPU serial program and GPU parallel programs. The results through comparison validation with the simulation of software FEKO are shown in Fig. 3., good agreement among them.
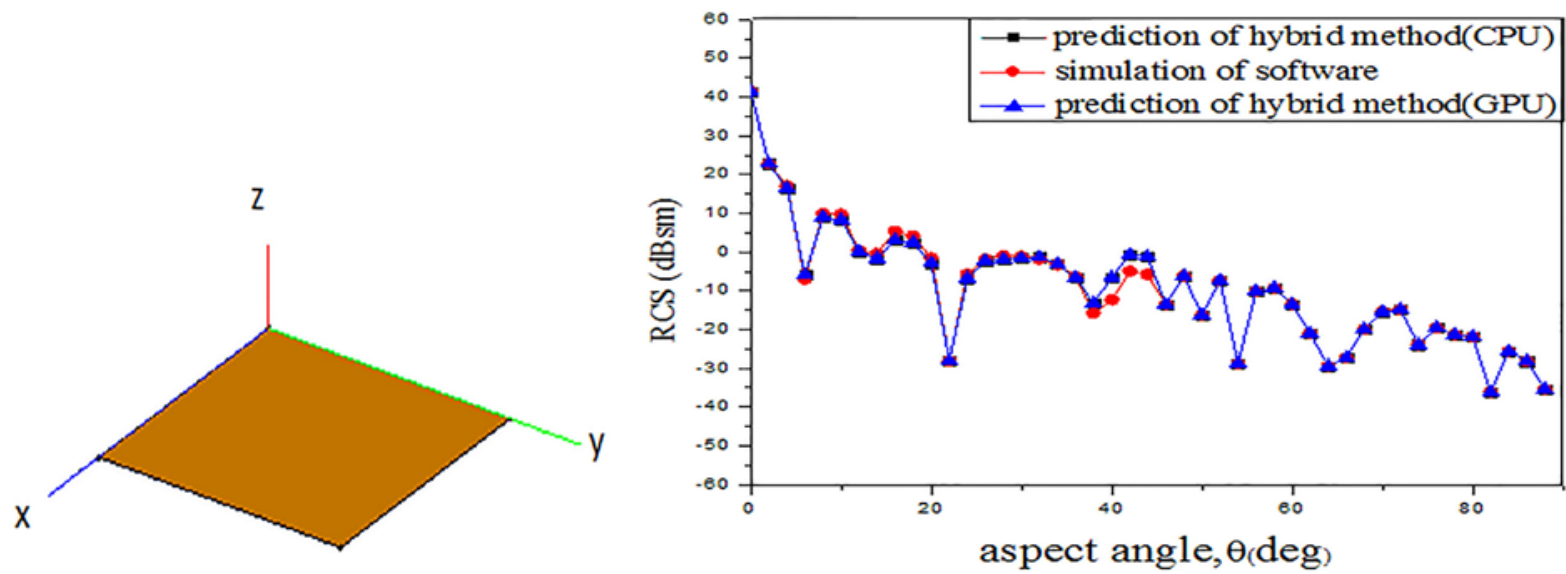

Fig. 2: The geometry model of $1 \mathrm{~m} * 1 \mathrm{~m}$ plate. Fig. 3: The simulation results of the plate RCS.

The next testing object is the geometry of a perfectly conducting rectangular prism model in free space and the rectangular prism size is $3 \mathrm{~m} * 0.6 \mathrm{~m} * 0.3 \mathrm{~m}$ (Fig. 4). Illuminated by a $300 \mathrm{GHz}$ plane wave, for HH-polarization is shown in Fig. 5, which shows a good agreement between the hybrid method result and FEKO-MOM result.

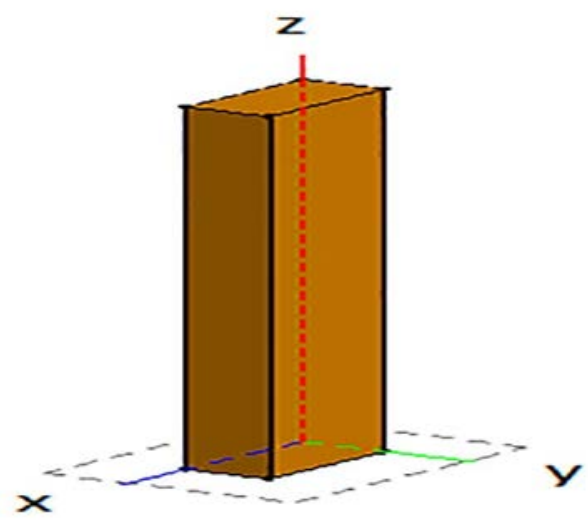

Fig. 4: The geometry model of the rectangular prism.

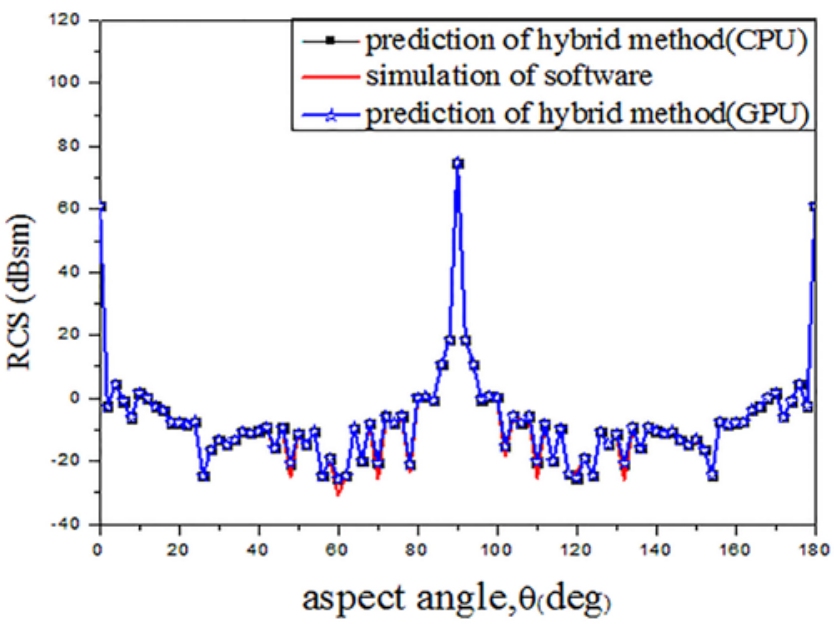

Fig. 5: Rectangular prism RCS comparison of simulation results.

In both the plate and rectangular prism, the GPU-Hybrid and CPU-Hybrid results are nearly identical to each other, it indicates that the GPU computation have no loss of accuracy.

To verify the capability and efficiency of the hybrid method, a simplified aerocraft is considered. The aerocraft (Fig. 6), is illuminated by plane waves with frequencies of $350 \mathrm{GHz}$. 

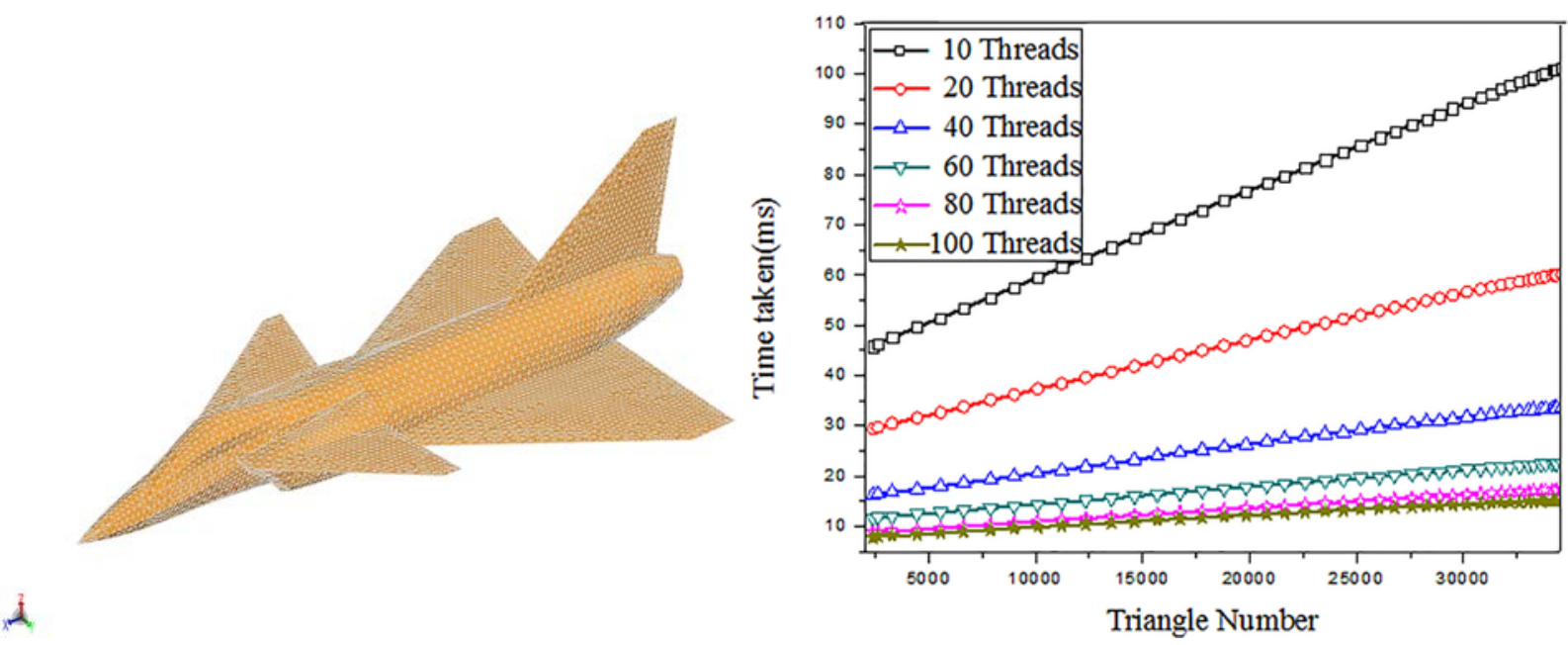

Fig. 6: The geometry model of the aerocraft. Fig. 8: Variation with thread counts of calculation time.

From Fig.7, it can be perceived that, the results solved by using GPU with CUDA parallel computing techniques and serial CPU program almost match perfectly. GPU time is the sum of data transfers time and kernel time. Thousands of the threads can be running simultaneously. To examine the performance improvement, the thread counts are gradually increased to achieve the parallel implementation of the solution algorithm using the GPU. Fig. 8 shows the execution times of the algorithm versus the number of threads in the model. It is found that the performance of the CUDA program is best when the thread size is increased.

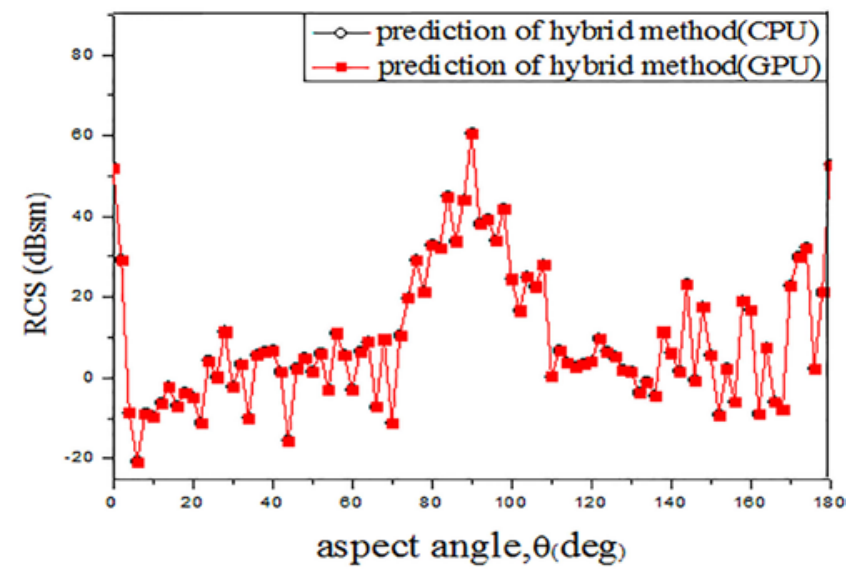

(a)

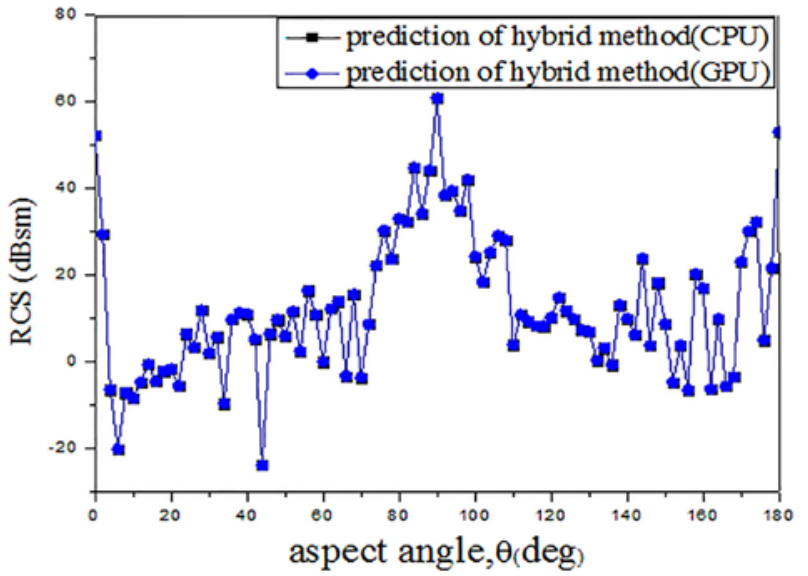

(b)

Fig. 7: The aerocraft RCS comparison of simulation of software FEKO, the hybrid method on

CPU and GPU. (a) HH-polarized monostatic RCS. (b) VV-polarized monostatic RCS

The speedup ratio is introduced to compare the CPU serial program and the GPU parallel program. The speedup ratios are shown in the Table. 1 for the hybrid method with different numbers of triangle facets. It can be seen that as triangular number increases, the speed-up ratio may also increase, and it is more obvious to the speedup effect.

Table 1. Computing time of GPU-Hybrid and CPU-Hybrid method

Triangular number $\quad$ GPU parallel program (ms) CPU serial program (ms) $\quad$ Speed-up ratio

\begin{tabular}{llll}
\hline 5550 & 1.9805 & 15 & 7.57 \\
10090 & 2.03824 & 31 & 15.2 \\
21632 & 2.17235 & 62 & 28.54 \\
22593 & 2.18419 & 78 & 35.71 \\
28262 & 2.24461 & 93 & 41.43 \\
\hline
\end{tabular}




\section{Conclusion}

A GRECO and MPO hybrid methods, which focuses on the diffraction fields of the target, is accelerated using GPU with the CUDA parallel programming model. The GPU-Hybrid method results are good agreement with the results of commercial software and CPU serial program. The GPU-Hybrid method has shown efficient to compute the diffraction fields. Compared with the CPU-based hybrid method, the GPU-Hybrid method can achieve great speedup with the aid of the GPU. Nevertheless, some slight discrepancies were observed, which is due to the creeping wave effects. In summary, the GPU-Based parallel hybrid method can easily be used for fast and efficient RCS prediction for the electrically large complex target.

\section{References}

[1] J.M. RIUS, M. Ferrando and L. Jofre: High-frequency RCS of complex radar targets in real-time. Sci. IEEE Antennas Propag.,Vol. 9 (1993), p. 1308.

[2] T. Shijo, L. Rodriguez and M. Ando: Accuracy demonstration of physical optics with modified surface-normal vectors, in: Processes in IEEE AP-S International Symposium and USNC/URSI National Radio Science Meeting, Albuquerque, NM (2006).

[3] Information on http://www.nvidia.com/object/cuda_home.html.

[4] J. Li, Y.H. Li and S.P. Chen: Fast image denoising with CUDA. Computer Engineering and App., Vol. 11 (2009), p.183.

[5] E.H. Wu: State of art and future challenge on general purpose computation by graphic processing unit. Sci. Journal of Soft., Vol. 10 (2004), p.1493.

[6] A.M. Svetlin: CUDA Compatible GPU as An Efficient Hardware Accelerator for AES Cryptography, in: Processes in IEEE International Conference on Signal Processing and Com, Dubai, United Arab Emirates, (2007).

[7] P.B.Wei, M. Zhang, W. Niu and W.Q. Jiang: GPU-based combination of GO and PO forelectromagnetic scattering of satellite. Sci. IEEE Trans. Antennas Propag., Vol. 11 (2012),p. 5278.

[8] J. Guan, S.Yan and J.M. Jin: An openMP-CUDA implementation of Multilevel Fast Multipole algorithm for electromagnetic simulation on Multi-GPU computing systems. Sci. IEEE Trans. Antennas Propag., Vol. 7 (2013), p. 3607.

[9] T. Tomasz, K. Andrzej and N. Artur: Using GPU with CUDA to accelerate MoM-Based electromagnetic simulation of wire-grid models. Sci. IEEE Trans. Antennas Propag. Let., Vol. 10 (2011), p.342.

[10]N. Tomoaki and W. Soichi: A GPU-based calculation using the three-dimensional FDTD method for electromagnetic field analysis, in: Processes in 32nd Annual International Conference of IEEE EMBS, Buenos Aires, Argentina (2010).

[11]T. Shijo and L. Rodriguez, Ando M.,: The modified surface-normal vectors in the physical optics. Sci. IEEE Trans. Antennas Propag., Vol. 12 (2008), p.3714. 